\title{
Antiplasticization in the Slightly Plasticized Poly(vinyl chloride)
}

\author{
Noriyuki Kinjo and Tsurutaro NAKAgAwa \\ Department of Polymer Science, Faculty of Science, Hokkaido University, \\ Sapporo, Japan. \\ (Received March 6, 1972)
}

\begin{abstract}
The dynamic mechanical moduli of slightly $(0-16 \mathrm{wt} \%)$ plasticized poly(vinyl chloride) were measured by the method of free damped oscillation in the temperature range from -180 to $+100^{\circ} \mathrm{C}$. The added plasticizers are tricresyl phosphate (TCP), butyl benzyl phthalate (BBP), dioctyl phthalate (DOP), dibutyl sebacate (DBS) and dioctyl sebacate (DOS). Two dispersion regions $(\alpha$ and $\beta)$ are observed in the case of poly(vinyl chloride) and they are influenced by addition of plasticizers. The $\alpha$-dispersion shifts to the lower temperature side and broadens with increasing plasticizer content. These effects are mainly dependent upon the plasticizer species, and of the above plasticizers their plasticizing ability decreases in the order of DOS $>$ DBS $>$ DOP $>$ BBP $>$ TCP. The $\beta$-dispersion, whose activation energy was estimated to be $14.3 \mathrm{kcal} / \mathrm{mol}$, first begins to disappear from the higher temperature side with increasing plasticizer content and ultimately vanishes. This effect scarcely depends upon the plasticizer species.

Young's moduli of these samples were measured at $-20-+40^{\circ} \mathrm{C}$ from observing the tensile stress relaxation. The modulus $v s$. plasticizer content curve shows a maximum. This is an example of "antiplasticization." The ability of additives to provoke antiplasticization decreases in the reverse order of plasticizing ability, that is, in the order of $\mathrm{TCP}>\mathrm{BBP}>\mathrm{DOP}>\mathrm{DBS}>$ DOS .

KEY WORDS Antiplasticization / Poly(vinyl chloride) / Plasticizer / Viscoelasticity /
\end{abstract}

The viscoelastic measurements of plasticized polymers provide many important clues for the elucidation of the mechanism of plasticization of polymers and the interaction between the polar polymer chain and plasticizers.

The case of slightly plasticized poly(vinyl chloride) (PVC) is interesting in that it constitutes not only a typical plasticized system in the usual sense, but also it behaves quite unusually in the glassy state below the glass transition temperature $T_{\mathrm{g}}$. That is to say, one observed (1) an increase of elastic modulus, (2) appearance of brittleness (a marked decrease in impact strength and ultimate elongation), (3) disappearance of the secondary $(\beta)$ dispersion, (4) invalidity of the additivity volume law, ${ }^{1,2}$ (5) decrease of the thermal coefficient of specific volume and (6) an anomalous behavior of the heat capacity, ${ }^{3}$ in PVC containing relatively small amounts of plasticizers.

Jackson and Caldwell ${ }^{4}$ observed that in some bisphenol A polycarbonates a marked increase of modulus and decrease of impact strength occur on addition (in a concentration up to 20\%) of several polar substances. They proposed a concept termed "antiplasticization" and discussed in detail these effects. The present case of PVC-plasticizer systems is an example of the antiplasticizing action with small amounts of platicizers. Studies of the mechanical and dielectric behavior of plasticized and unplasticized PVC have been made by several workers. ${ }^{2,5-14}$ Ghersa $^{5}$ and Jacobson ${ }^{6}$ observed that the modulusenhancing effect of additives is in the same order of their effect on the decrease in impact strength and ultimate elongation. Fuoss, ${ }^{7}$ in his dielectric experiment, observed the disappearance of the $\beta$-dispersion in PVC plasticized with up to $20 \%$ diphenyl. Bohn ${ }^{8}$ carried out viscoelastic measurements on slightly plasticized PVCs and found that the mechanical $\beta$-dispersion was very much reduced by the presence 
of small amounts of plasticizers, in accord with the dielectric results by Fuoss. He discussed the appearance of brittleness in relation to the disappearance of the $\beta$-dispersion. Fujii ${ }^{1}$ uncovered the antiplasticization in the PVC-NBR (acrylonitrile-butadiene rubber) blend systems. $\mathrm{He}$ discussed this phenomenon from the viewpoint of compatibility between PVC and NBR.

The molecular mechanism of the antiplasticization or the decrease of the $\beta$-dispersion has been discussed by various authors. Many workers, including Jackson, et al., ${ }^{4}$ and Pezzin, et al. ${ }^{9}$ assume simple cohesion and hindrance of local motion by polar interactions. Mikhailov, et al., ${ }^{10,11}$ studied the dielectric dispersion and IR spectra of plasticized PVC, and concluded that the decrease of the dielectric $\beta$-dispersion is related to a change in conformation of the PVC molecules. Robeson ${ }^{2}$ and Faucher ${ }^{15}$ considered that the antiplasticization is attributable to the filling of polymer free volume and that the molecular motion responsible for the lowtemperature transition is restricted.

Kakutani, et al. ${ }^{38}$ reported that the $\beta$-dispersion of PVC is composed of two dispersions and concluded that the one is due to the molecular motion in the crystalline part and the other due to that in the amorphous part of PVC. On the basis of Kakutani's results, the authors previously proposed a "two phase" model. ${ }^{37}$ Each plasticizer was assumed to differ in its solvating power; some intrudes into the amorphous part only, and others into both the amorphous and crystalline parts, etc. The relation between plasticization and antiplasticization in PVC was interpreted in terms of this specificity of the plasticizer. There was observed, however, no such multiple $\beta$-dispersion in the present study, and therefore a new interpretation is proposed herein.

In this paper we report the experimental results on stress relaxation, dynamic-mechanical properties and temperature dependence of specific volume for unplasticized and plasticized PVC. It is the purpose of the present investigation to elucidate the mechanism of antiplasticization while paying special attention to the difference of the diluent species.

\section{EXPERIMENTAL}

\section{Samples}

Commercial PVC, a product of Denki Kagaku Chemical Industries Co., Ltd., having an average degree of polymerization $P$ 1100, was used and this PVC powder was blended with variable amounts of plasticizers and small amounts of stabilizers, calendered by a hot roller for $10 \mathrm{~min}$ at $170^{\circ} \mathrm{C}$, and compression-molded into sheets $0.5 \mathrm{~mm}$ and $5 \mathrm{~mm}$ in thickness. The plasticizers added were all commercial materials: tricresyl phosphate (TCP), butylbenzyl phthalate (BBP), dioctyl (di-2-ethylhexyl) phthalate (DOP), dibutyl sebacate (DBS), and dioctyl (di-2-ethylhexyl) sebacate (DOS). The plasticizer contents are $4.5,8.7,12.5$, and $16.0 \mathrm{wt} \%$. In order to remove the residual stress $^{16}$ introduced in the process of molding, all samples were used after annealing with a mean rate of temperature decrease of $0.2^{\circ} \mathrm{C} / \mathrm{min}$ from $120^{\circ} \mathrm{C}$ to room temperature. In addition, samples quenched from $120^{\circ} \mathrm{C}$ to liquid nitrogen temperature were used to investigate the effect of thermal treatments.

\section{Measurements}

The measurements of the complex shear modulus of elasticity were carried out by the method of free damped torsional oscillation with the strip-shaped specimens $(0.5 \times 7 \times 70$ and $8 \times 4 \times 70 \mathrm{~mm})$ at frequencies of $3.5-5 \mathrm{~Hz}$ in the temperature range from $-180^{\circ} \mathrm{C}$ to $+100^{\circ} \mathrm{C}$. The storage shear modulus $G^{\prime}$, the loss shear modulus $G^{\prime \prime}$, and the loss $\operatorname{tangent} \tan \delta$ were derived by well-known equations. ${ }^{17}$

Tensile stress relaxation in a rod-like sample (10-cm length and $0.06-\mathrm{cm}$ diameter) under constant tensile strain was observed by using a strain gauge and an amplifier recorder.

The temperature dependence of sfecific volume was examined by means of dilatometer with the usual procedure. ${ }^{18}$

\section{RESULTS AND DISCUSSION}

\section{Glass Transition Region}

The authors reported previously ${ }^{17}$ the effects of some plasticizers on the mechanical properties of PVC in the glass-transition region, including dilatometric results. The results showed that the glass-transition temperature $T_{\mathrm{g}}$ obtained by 
Antiplasticization in the Slightly Plasticized PVC

dilatometry decreases monotonously with the increase of plasticizer content and that at the same time the relaxation mechanism of glasstransition (or $\alpha$-dispersion) region was influenced by the addition of plasticizers. The temperature dependence of the shift factor $a_{\mathrm{T}}$ obtained from the stress relaxation deviates from the WLF equation. The temperature dependence of the apparent activation energy decreases. The master curves of the relaxation moduli flatten and shift to a shorter time region, and the broadening of the temperature dispersion curves of dynamic

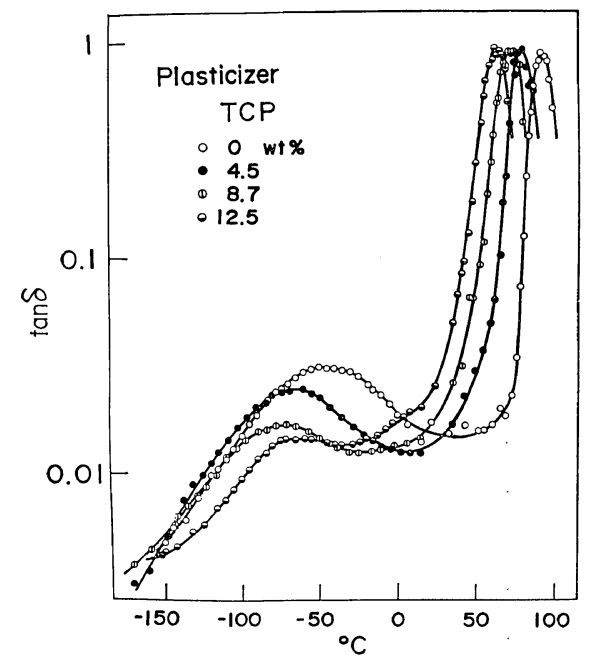

Figure 1. Effect of plasticization on the loss tangent of PVC.

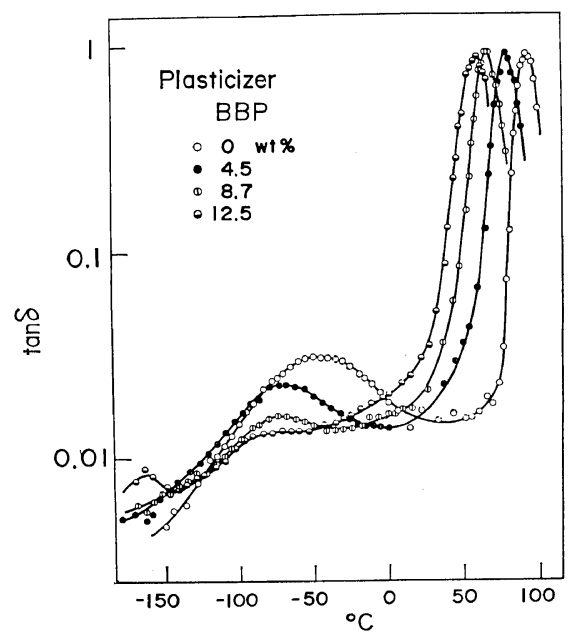

Figure 2. Effect of plasticization on the loss tangent of PVC.

Polymer J., Vol. 4, No. 2, 1973 moduli occurs. Moreover, it should be emphasized that the tendency of decreasing $T_{\mathrm{g}}$ and of affecting the relaxation mechanism greatly depends upon the plasticizer species. In summary, the order of plasticizing ability is as follows.

$$
\text { DOS }>\text { DBS }>\text { DOP }>\text { BBP }>\text { TCP } \text {. }
$$

In the present study, dynamic-mechanical measurements were carried out with unplasticized and plasticized PVC over a wide temperature range $-180-+100^{\circ} \mathrm{C}$ at low frequencies (period, 3-5

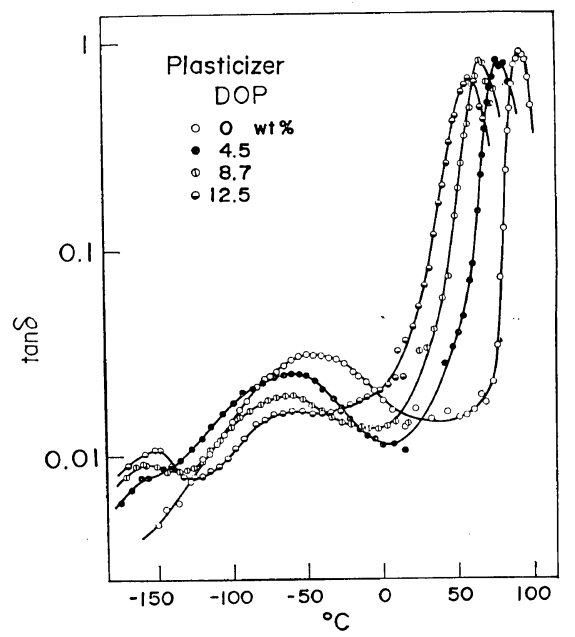

Figure 3. Effect of plasticization on the loss tangent of PVC.

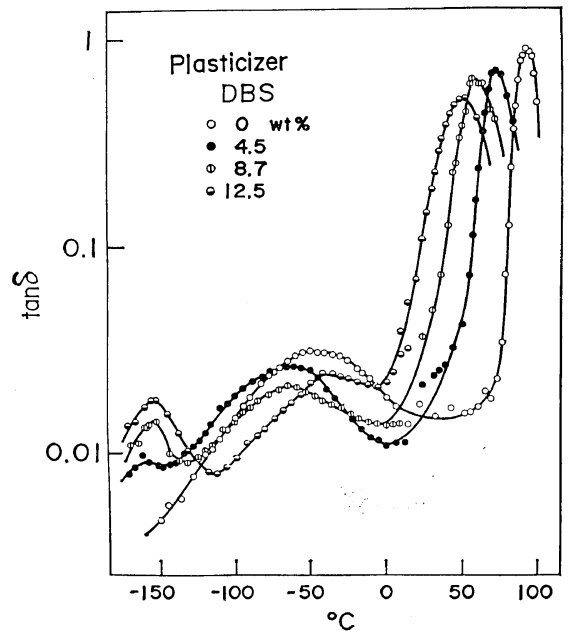

Figure 4. Effect of plasticization on the loss tangent of PVC. 


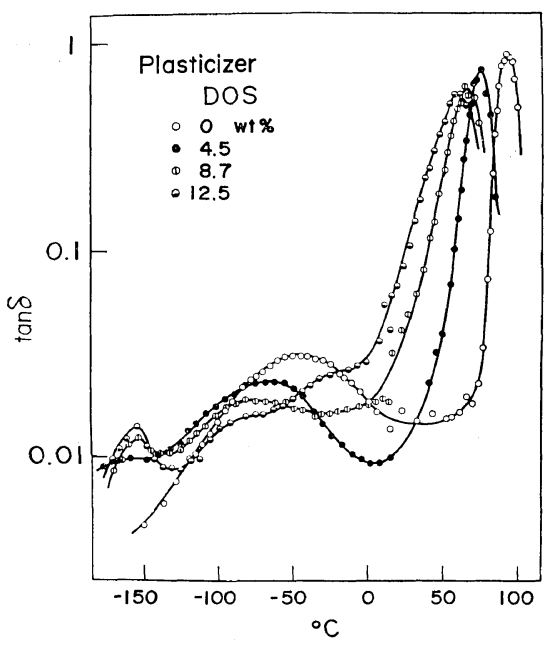

Figure 5. Effect of plasticization on the loss tangent of PVC.

sec) with the aim of comparing the effects of various plasticizers. Two dispersion regions exist in the case of PVC, and they are affected to a great extent by the addition of plasticizers as illustrated in Figures $1-5$ where the values of $\tan \delta$ are plotted against temperature at varying plasticizer content. The one at higher temperatures (glass transition or $\alpha$-dispersion) shifts to a lower temperature with the increase of plasticizer content, the height of the maximum lowers, and the peak widens. These effects are observed more markedly in the systems containing more effective plasticizers (DOS and DBS) than in the systems containing less effective ones (TCP and BBP). The other dispersion at lower temperatures, the so-called secondary or $\beta$-dispersion, is attributed to "the small scale motions of small segments of the main chain," termed "local relaxation mode,",19 and this $\beta$-dispersion is markedly reduced by the presence of plasticizers.

A new dispersion is observed at about $-155^{\circ} \mathrm{C}$ in PVC samples plasticized with DOP, DBS and DOS, whereas this dispersion is not entirely observed in the PVC-TCP system. The details of the $\beta$-dispersion and the new dispersion will be described later.

\section{Low Temperature Region}

In Figure 6, values of loss modulus $G^{\prime \prime}$ are plotted as a function of the radian frequency at

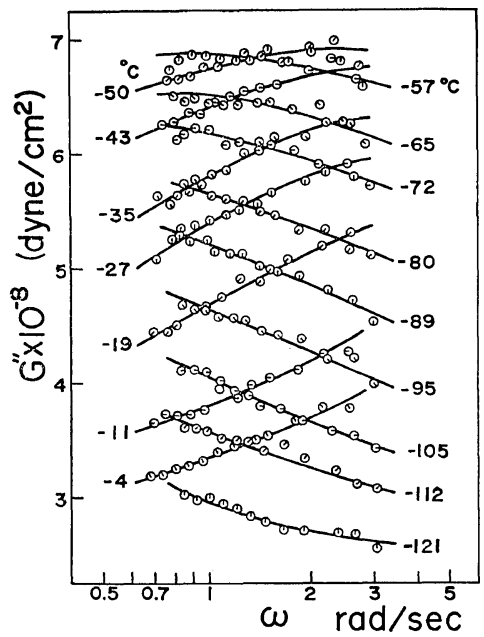

Figure 6. Radian frequency dependence of the shear loss modulus, $G^{\prime \prime}$, relating to the $\beta$-dispersion of PVC.

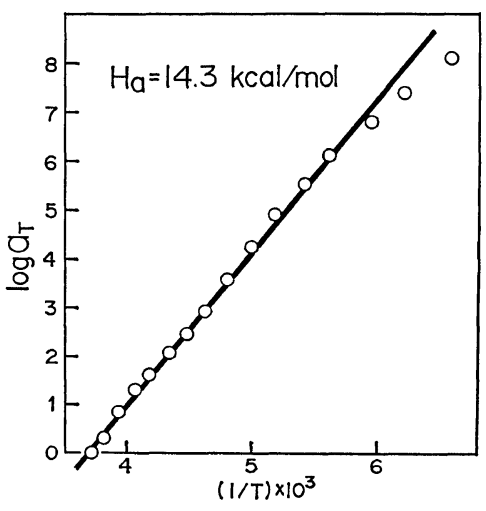

Figure 7. Logarithms of the shift factor, $\log a_{\mathrm{T}}$, $v s$. reciprocal absolute temperature, $1 / T$.

different temperatures which cover the $\beta$-dispersion region of unplasticized PVC. A plot of the logarithm of the shift factor, $\log a_{\mathrm{T}}$, against reciprocal temperature, as shown in Figure 7, conforms to the Arrhenius-type relationship, while the data for the $\alpha$-dispersion fit the WLF equation. ${ }^{17}$ The apparent activation energy $\Delta H_{\mathrm{a}}$ for the $\beta$-dispersion has been estimated to be $14.3 \mathrm{kcal} / \mathrm{mol}$, which is analogous to the values $15.7 \mathrm{kcal} / \mathrm{mol}$ obtained by Rnåby and 13.5 $\mathrm{kcal} / \mathrm{mol}$ by Bohn. $^{8}$ Figure 8 shows the composite curves obtained by plotting the data of Figure 7 with the method of reduced variables and by reducing to $-4^{\circ} \mathrm{C}$. The com- 


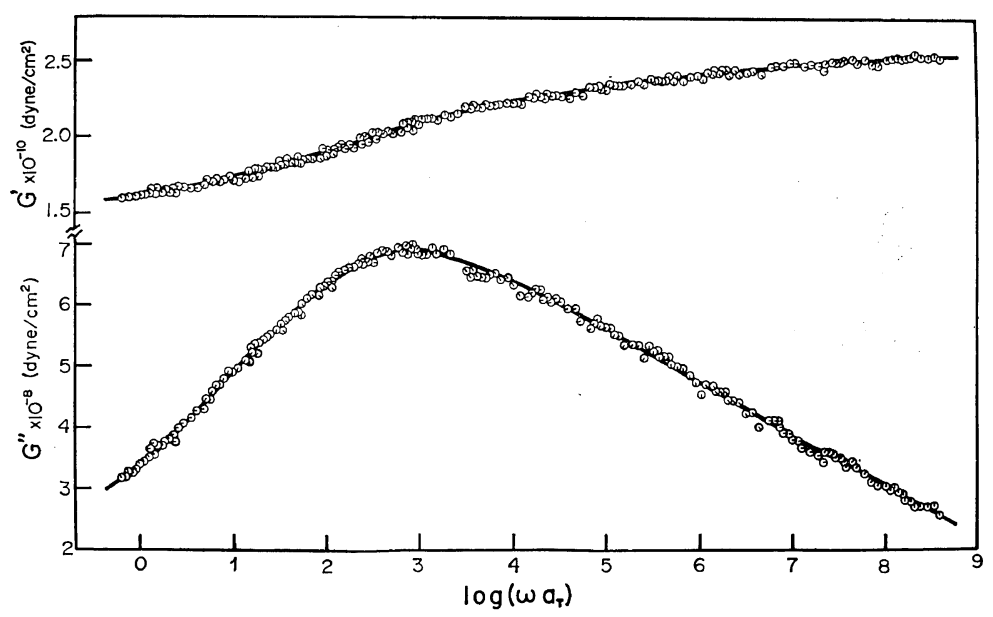

Figure 8. Composite relaxation curves of real and imaginary parts of complex rigidity, reduced to $-4^{\circ} \mathrm{C}$.

posite curve of loss modulus $G^{\prime \prime}$ gives, as a first approximation, the distribution of the relaxation times. The composite curves in Figure 8 suggest that the spectrum of $\beta$-dispersion of PVC is very broad and unsymmetrical about its peak, being steeper on the longer relaxation time side. This result coincides with the general description of the local relaxation mode of polymers without side chains. ${ }^{19}$

The term "antiplasticization" has been ascribed to the mechanical behavior observed by addition of certain low-molecular-weight additives to polymers with an increase in modulus and appearance of brittleness. ${ }^{4}$

Results of the tensile test are shown in Figure 9 in which the relaxation Young's modulus after $10 \mathrm{sec}, E_{\mathrm{r}}(10)$, is plotted against plasticizer content at various temperatures. The ten-second modulus curves show a maximum which shifts to lower concentrations with the increase of temperature and finally disappears. In the cases of DOS and DBS, described above as effective plasticizers, the increase of $E_{\mathrm{r}}(10)$ by addition of these diluents is very slight and the $E_{\mathrm{r}}(10)$ peak is situated at about $5 \mathrm{wt} \%$; at higher plasticizer concentrations the normal softening effect is observed. In the case of less effective plasticizer as TCP, the increase of $E_{\mathrm{r}}(10)$ is more remarkable and the modulus-enhancing effect by TCP observed over fairly higher TCP concentrations. The ability of plasticizers to provoke anti-

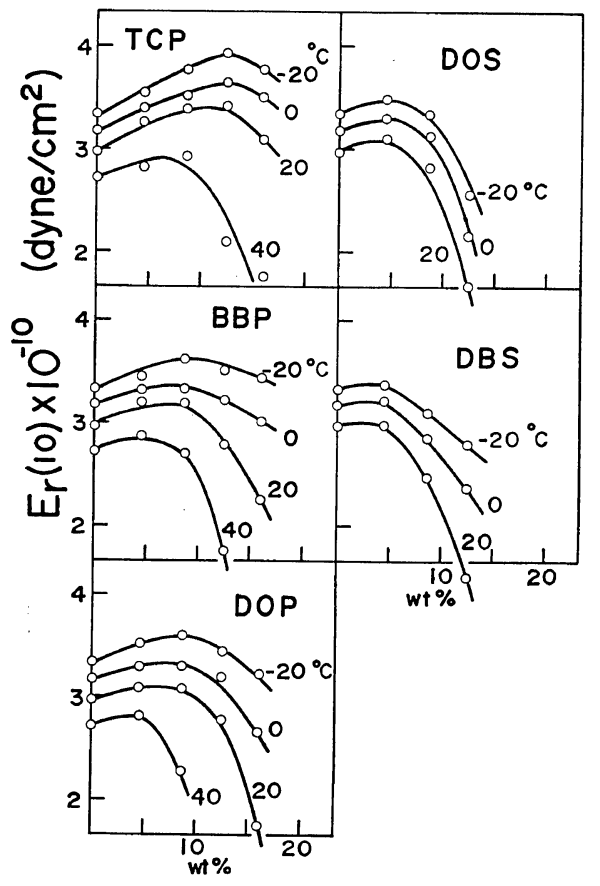

Figure 9. Effect of various plasticizers on the ten-second modulus, $E_{\mathrm{r}}(10)$, plotted against plasticizer content.

plasticization is concluded from the Figures to be in the order of

$$
\mathrm{TCP}>\mathrm{BBP}>\mathrm{DOP}>\mathrm{DBS}>\mathrm{DOS} \text {. }
$$

This order is in reverse relation to the plasticiz- 


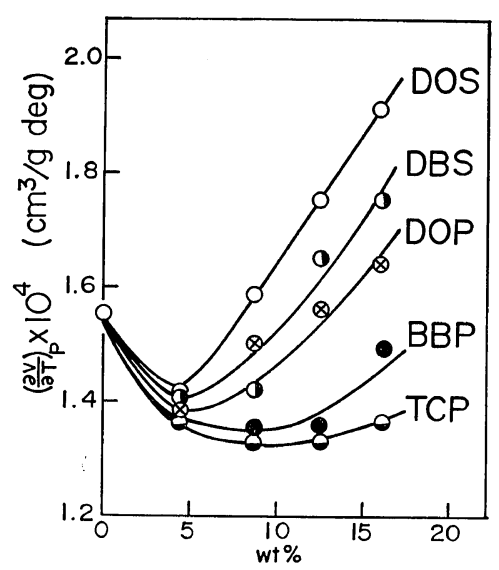

Figure 10. Temperature coefficients of specific volume of the PVC-plasticizer systems plotted against plasticizer content (wt \%).

ing ability. Ghersa ${ }^{5}$ and Jacobson ${ }^{6}$ reported that the appearance of brittleness was observed in the parallel order with the modulus-enhancing effect. At any rate, it is noteworthy that the antiplasticizing effect greatly depends upon the additive species.

In Figure 10, the temperature coefficient of the specific volume obtained from the dilatometry in the temperature range, $-20-+50^{\circ} \mathrm{C}$, is plotted against plasticizer content. The temperature coefficients of specific volume of DOP and TCP were reported to be $7.7 \times 10^{-4}$ and $5.8 \times 10^{-4} \mathrm{~cm}^{3} / \mathrm{g}$ deg respectively in the same temperature range. ${ }^{21}$ The other plasticizers are assumed to have comparative values, which are larger than the value, $1.55 \times 10^{-4} \mathrm{~cm}^{3} / \mathrm{g} \mathrm{deg}$, of PVC in the glassy state. The results in Figure 10 are particularly remarkable in that the temperature coefficient of specific volume of the PVC-plasticizer systems is lower than any of the value of unplasticized PVC and those of pure diluents as long as the plasticizer content is small.

It can be considered that the temperature coefficient of specific volume or the thermal expansion coefficient are closely related to the molecular interaction or the cohesive energy density. Therefore this anomaly in the PVCplasticizer systems is interpreted by considering that the addition of small quantity of plasticizer to PVC in the glassy state brings about the increase of cohesive energy density. The enhancement of cohesion is in the order of

$$
\text { TCP }>\text { BBP }>\text { DOP }>\text { DBS }>\text { DOS } \text {. }
$$

This order is in agreement with the order of antiplasticizing ability.

Tobolsky $^{22}$ reported that the poly(methyl methacrylate) (PMMA) sample preconditioned by cooling rapidly from above the $T_{\mathrm{g}}$ exhibits a rapid relaxation of stress, but a sample cooled slowly shows a very much slower relaxation of stress. Quoting Tobolsky's result, Boyer $^{23}$ pointed out that the amount of free volume in the glassy state of amorphous polymer can be controlled by the thermal treatment, and the physical properties of polymer below $T_{\mathrm{g}}$ can vary significantly as a consequence of this effect. Hence, Boyer proposed a concept termed "degree of glassiness" which arises from varying amounts of free volume below $T_{\mathrm{g}}$. However, Pezzin, et al. ${ }^{9}$ reported that the $\beta$-peak of PVC is not affected by a change of density (that is of free volume) caused by thermal treatments. The results of viscoelastic studies of annealed samples under the cooling rate $0.1^{\circ} \mathrm{C} / \mathrm{min}$ and quenched samples cooled rapidly from $120^{\circ} \mathrm{C}$ to liquid nitrogen temperature are shown in Figures 11

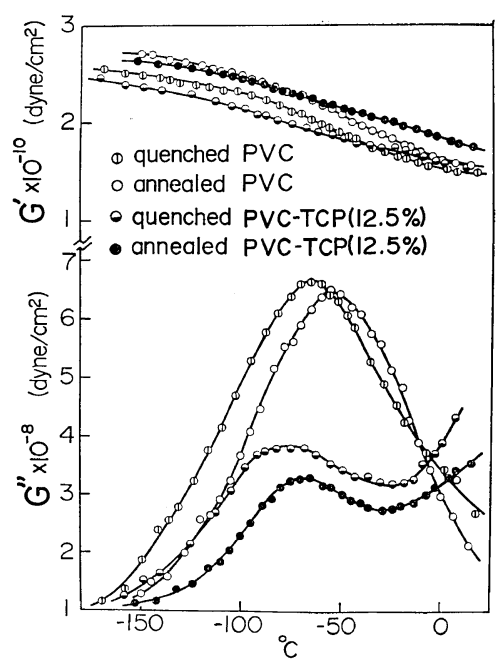

Figure 11. Effect of thermal treatment on the $\beta$ dispersion region. The real and imaginary parts of complex rigidity of quenched and annealed PVC, and of quenched and annealed PVC-TCP $(12.5 \mathrm{wt} \%)$ systems are plotted against temperature. 


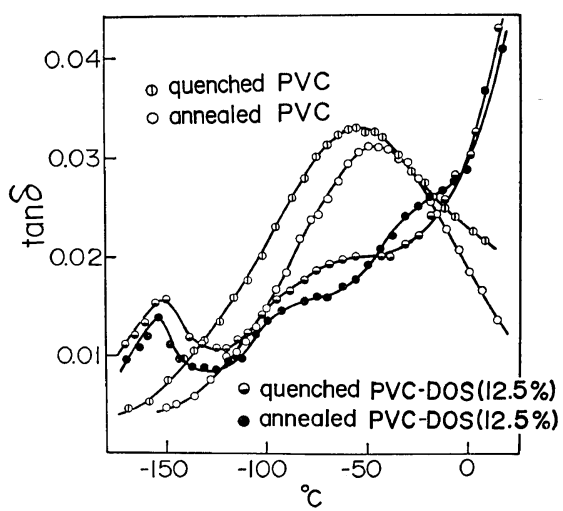

Figure 12. Effect of thermal treatment on the $\beta$ dispersion region. The loss tangents of quenched and annealed PVC, and of quenched and annealed PVC-DOS (12.5 wt \%) systems are plotted against temperature.

and 12 , in which $G^{\prime}, G^{\prime \prime}$, and $\tan \delta$ are plotted against temperature on a linear scale. The modulus of the quenched samples is lower than that of the annealed samples. The $\beta$-dispersion region shifts to lower temperatures and the height of the $\beta$-peak becomes higher by quenching.

The effect of pressure on the $\beta$-peak has been examined by some authors. ${ }^{24-26}$ They found that the $\beta$-peak becomes smaller and shifts to a lower frequency region with increasing the

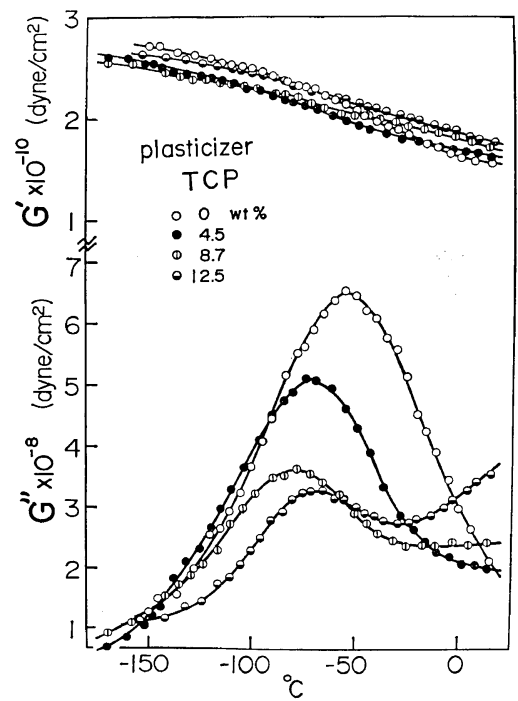

Figure 13. Effect of plasticization on the $\beta$-dispersion of PVC. pressure, that is, with a decreasing amount of free volume. It may therefore be concluded that the increase of the amount of free volume facilitates the relaxation in the glassy state, because of decreasing the internal friction.

In PVC composition plasticized with DOS (12.5 wt $\%$ ), a new shoulder on the $\tan \delta$ vs. temperature curve was observed near $-30^{\circ} \mathrm{C}$, but this shoulder disappears by quenching as illustrated in Figure 12. The details about this shoulder are not clear at present.

From the fact that the observed density of polymer-diluent systems in the glassy state is higher than the density for these systems calculated from the simple additivity rule, some authors $^{1,2,4,27}$ concluded that the diluent added initially fills polymer free volume. However, the effect of the decrease of polymer free volume on the $\beta$-peak by addition of diluent is not identical with that of the decrease of polymer free volume by thermal history or pressure. This is understood from the fact that the $\beta$-peak shifts to lower temperatures (to higher frequency region) by the addition of plasticizers in contrast to the case of thermal treatment, etc., in which the $\beta$-peak shifts to higher temperatures (to lower frequency region).

In Figures 13-17, the shear storage modulus $G^{\prime}$ and shear loss modulus $G^{\prime \prime}$ obtained for various plasticized systems are plotted against

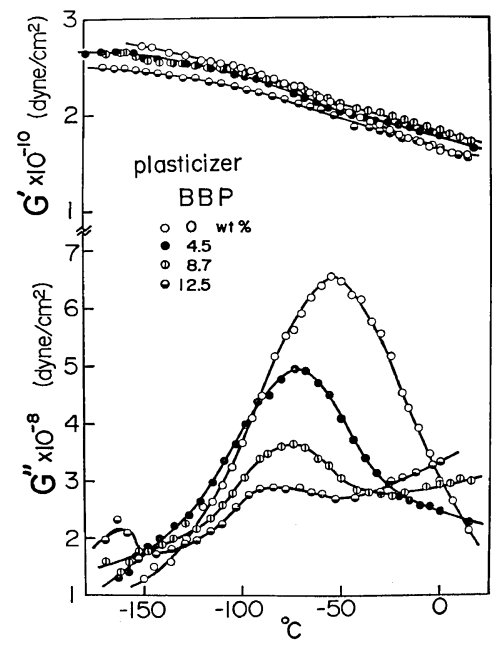

Figure 14. Effect of plasticization on the $\beta$-dispersion of PVC. 


\section{N. Kinjo and T. NAKagawa}

temperature on a linear scale. Again antiplasticization is observed in these results, coinciding with the results of tensile tests; above the $\beta$-transition temperatures (above $-50^{\circ} \mathrm{C}$ ), an increase of $G^{\prime}$ by addition of diluents is observed. However, at the lower temperatures (under $-70^{\circ} \mathrm{C}$ ) the modulus of plasticized PVC is lower than that of unplasticized one. This is attributable to the decrease of the cohesive energy density due to the substitution of unbonded solvent molecules for covalently bonded polymer segments.

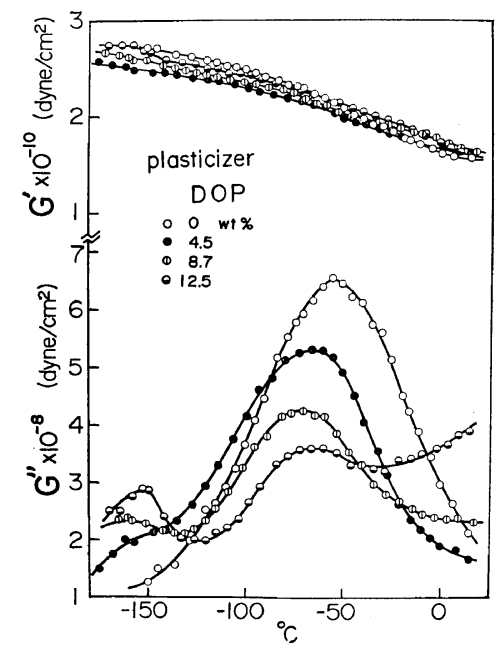

Figure 15. Effect of plasticization on the $\beta$-dispersion of PVC.

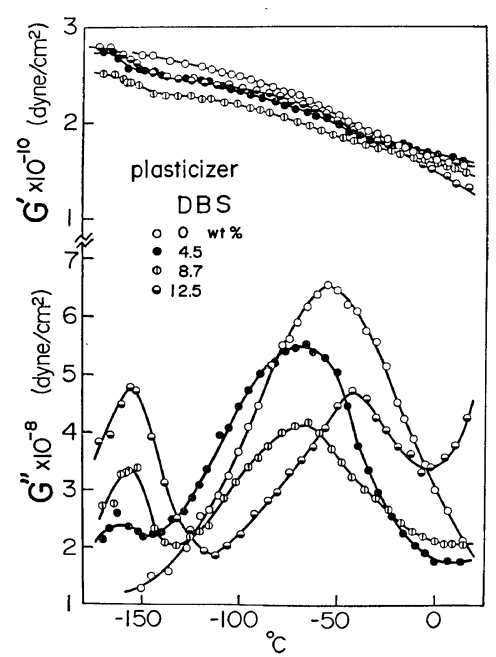

Figure 16. Effect of plasticization on the $\beta$-dispersion of PVC.
The $\beta$-dispersion is suppressed with the increase of plasticizer content and ultimately vanishes. Since a drop in shear modulus occurs when passing through mechanical loss transition, the elimination of this transition results in the elimination of any noticeable drop in modulus. This result predicts a higher modulus above the transition temperature. ${ }^{2}$ The overall effect of the plasticizer on the $\beta$-dispersion can be described from Figures $13-17$ as reducing the local motion of the chain segments responsible for the mechanical absorption. Furthermore it is noteworthy that the $\beta$-dispersion is eliminated gradually from the higher temperature side, that is, from the longer relaxation time side. Wada, et al. ${ }^{28,29}$ reported the relationship between the relaxation strength of local mode dispersion and the number of units in a co-operatively vibrating segment. At the present stage, however, no plausible theory about the distribution of relaxation times of local mode exists, and the authors tentatively interpret the experimental results as follows. The local motion of relatively longer segments corresponding to the longer relaxation time is at first suppressed by addition of plasticizers. The dependence of this effect on the nature of the plasticizer is less evident. The intrusion of the $\alpha$-dispersion is more notable in the systems containing effective plasticizers (DBS and DOS)

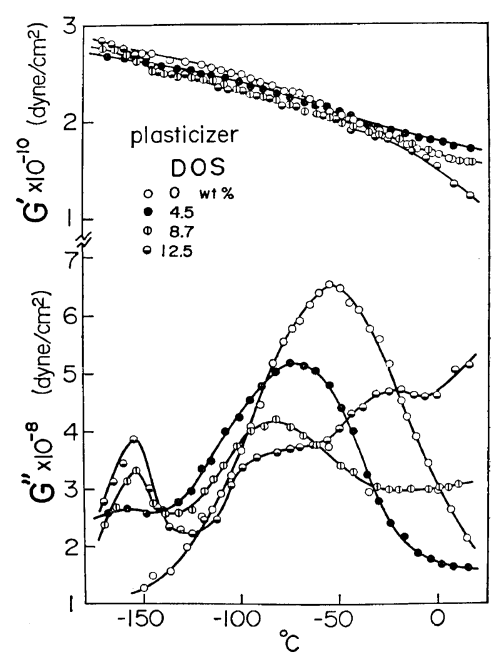

Figure 17. Effect of plasticization on the $\beta$-dispersion of PVC. 
than in the systems containing less effective ones (TCP and BBP).

As illustrated in Figures 13-17, a new dispersion was observed near $-155^{\circ} \mathrm{C}$ in $\mathrm{PVC}-$ DOP, PVC-DOS, and PVC-DBS systems in which the molecular structure of plasticizer is characteristic in having long alkyl chains. But this dispersion is hardly observed in the PVCBBP system and entirely absent in the PVCTCP system. This new absorption becomes larger with increasing plasticizer content. Therefore it may be admissible to consider this newly detected dispersion to be due to the molecular motion in the alkyl chains of plasticizers. Perhaps this dispersion is identical in nature with one of the $\gamma$-dispersions in polyethylene which is considered to be associated with the motion of short chain of $\mathrm{CH}_{2}$ groups in amorphous region. ${ }^{30,31}$

\section{INTERPRETATION OF THE ANTI- PLASTICIZATIONN}

On the basis of the results obtained above, the antiplasticization and the relating phenomena in the slightly plasticized PVC would be interpreted as follows.

In general, a mechanical transition which accompanies a drop in elastic modulus and a maximum of loss modulus is observed when some molecular motion occurs. If this molecular motion is restrained by some effects, the mechanical transition will not be observed. In this case no noticeable drop in elastic modulus has to occur, resulting in an unchanged modulus above the transition temperature, and the maximum of loss modulus must decrease. Slightly plasticized PVC in the glassy state is a typical example which shows the above-mentioned phenomena. In this system the polar plasticizer molecules cohere to PVC segment on account of their dipole-dipole interaction and the motion of cohering parts of PVC chain is extremely hindered, that is, the PVC chain is locally cemented at these positions. Therefore it might be considered that at first the local mode motion of PVC chain will be restrained starting from the ones of the relatively longer segments. The result is that the $\beta$-dispersion is suppressed from the higher tempera- ture side with increasing plasticizer content and at the same time an increase of modulus (antiplasticization) is observed. In relation to this, moreover, the disappearance of the $\beta$-dispersion brings about the effect that a ductile folymer changes into a brittle one, as Bohn suggested. ${ }^{8}$ Some authors, ${ }^{32-35}$ including Bohn, uncovered a relationship between secondary dispersion and impact strength, and proposed that the systems having a larger secondary dispersion exhibit a higher impact strength and that the impact strength increases above the secondary transition temperature. Bohn postulates that the decrease of impact strength on adding small amounts of plasticizer is related to the disappearance of the $\beta$-dispersion.

The reason why the temperature coefficient of specific volume of PVC-plasticizer systems shows a lower value than that of unplasticized PVC, as observed in Figure 10, is explained as follows. Heydemann, et al. ${ }^{36}$ reported that two bend points, $\mathbf{A}$ and $\mathbf{B}$, are observed in the specific volume-temperature curve of PVC. It is considered that the one at higher temperature, $\mathrm{A}$, is attributable to the glass transition and the other, $\mathbf{B}$, to the $\beta$-dispersion. In our case of slightly plasticized PVC in which the $\beta$-dispersion is eliminated, point $B$ disappears, and the value of the temperature coefficient of specific volume remains constant over the entire temperature range below the $T_{\mathrm{g}}$. Thus the additivity law of volume is considered invalid in this case.

According to our results, the suppressing effect on the $\beta$-dispersion scarcely depends upon the plasticizer species. This means that there is no appreciable difference from the viewpoint of the ability of the local cementation of the molecular chains. On the basis of this result, it would duly be expected that all additives would act similarly for antiplasticization. However, as shown in Figure 9, the antiplasticizing ability of these additives differs distinctly in contrast with this expectation. In order to interpret these features, attention was focused on the fact that the relaxation mechanism of the $\alpha$-dispersion is influenced by addition of plasticizers and that this effect much depends upon the nature of the additives.

A less effective plasticizer as TCP is not so effective in reducing $T_{\mathrm{g}}$ and broadening the $\alpha$ - 


\section{N. KINJo and T. NAKAGAwA}

dispersion. In the PVC-TCP system, the mechanical transition due to the glass transition occurs in a narrow temperature range; i.e, a rapid drop in elastic modulus and a steep loss maximum are observed. ${ }^{17}$ In this case the restraining effect of TCP on the local mode motion and on the mechanical properties is straightforward. That is to say, a drop in modulus which occurs on passing through the $\beta$-transition becomes smaller resulting in comparatively higher modulus above this transition temperature, and at the same time the appearance of brittleness is observed because of the reduction of loss maximum.

In contrast to this, the case of DOS and DBS is a little complicated. In the PVC specimens plasticized with effective plasticizers (DOS and DBS), the mechanical transition due to the glass transition is observed over a very wide temperature range ${ }^{17}$; the modulus begins to decrease from the fairly low temperature and shows a slow decrease with a rise in temperature; the loss peak is flattened and spreads over a wide range, and the intrusion of the $\alpha$-dispersion into the lower temperature region becomes remarkable as shown in Figures 16 and 17. In this case, the increase of the elastic modulus which should be caused by the restraint of the local mode motion is cancelled out by the modulus-decreasing effect of the intruding $\alpha$ dispersion. The antiplasticization is therefore very slight. The impact strength is not expected to show a low value, because of the intrusion of the $\alpha$-dispersion (and perhaps because of the dispersion near $-155^{\circ} \mathrm{C}$ ) which is assumed to serve as an energy absorbing mechanism in the impact test in place of $\beta$-mechanism.

Consequently, even though a serious difference among the nature of the plasticizer is not observed in the effect on the elimination of the $\beta$-dispersion, the antiplasticizing ability of additives appears rather diverse owing to the fact that the added plasticizers behave fairly specifically in the $\alpha$-dispersion region. The apparently reverse relation between the antiplasticizing ability and the usual plasticizing ability of additives is thus properly understood from the above-mentioned viewpoint.

Acknowledgement. The authors wish to thank Dr. M. Kikkawa and Mr. S. Kawada of the
Hitachi Electric Co., Ltd., for supplying samples. The authors also wish to express their gratitude to Associate Professor T. Komatsu and Mr. K. Nakamura of Hokkaido University for their encouragement and discussions during the course of the present study. The present work has been supported in part by Grant for Scientific Research from the Ministry of Education.

\section{REFERENCES}

1. H. Fujii, Aichiken Kogyoshidosho Hokoku (Reports of Research Institure, Aichi Prefecture), (5), 67 (1969).

2. L. M. Robeson, Polym. Eng. Sci., 9, 277 (1969).

3. L. Dunlap, J. Polym. Sci., Part C, 30, 561 (1970).

4. W. J. Jackson and J. R. Caldwell, J. Appl. Polym. Sci., 11, 211, 227 (1967).

5. P. Ghersa, Modern Plastics, 36, 135 (1958).

6. U. Jacobson, Brit. Plastics, 32, 152 (1959).

7. R. M. Fouss, J. Amer. Chem. Soc., 63, 369 (1941).

8. L. Bohn, Kunststoffe, 53, 826 (1963).

9. G. Pezzin, G. Ajroldi, and D. M. Garbuglio, J. Appl. Polym. Sci., 11, 2553 (1967).

10. G. P. Mikhailov, A. M. Lovanov, and D. M. Mirkamilov, Vysokomol. Soedin., 8, 1351 (1966).

11. G. P. Mikhailov, D. M. Mirkamilov, Yu. Ya. Gotlib, A. M. Lobanov, and B. Z. Volchek, ibid., Ser. A, 9, 1967 (1967).

12. M. Okuyama and T. Yanagida, Kobunshi Kagaku (Chem. High Polymers), 20, 385 (1963).

13. M. Matsuo, T. Nozaki, and Y. Jho, J. Electron Microscopy, 17, 7 (1968).

14. J. Malác, V. Altmann, and J. Zelinger, $J$, Appl. Polym. Sci., 14, 161 (1970).

15. L. M. Robeson and J. A. Faucher, J. Polym. Sci., Part B, 7, 59 (1969).

16. S. Saito, Repts. Progr. Polym. Phys. Japan, 11, 375 (1968).

17. N. Kinjo, T. Komatsu, and T. Nakagawa, Nippon Kagaku Zasshi (J. Chem. Soc. Japan), 92, 27 (1971).

18. I. Uematsu in "Zikken Kagaku Koza (Hand Book of Experimental Chemistry),"' Vol. 8, The Chemical Society of Japan, Ed., Maruzen Publishing Co. Ltd., Tokyo, 1957, p 83.

19. N. Saito, K. Okano, S. Iwayanagi, and T. Hideshima, "Solid State Physics," Vol. 14, F. Seitz and D. Turnbull, Ed., Academic Press, New York, N. Y., 1963, p 387.

20. J. Petersen and B. Rånby, Macromol. Chem., 133, 251 (1970). 
21. Unpublished data obtained by T. Takahashi.

22. J. R. McLoughlin and A. V. Tobolsky, $J$. Polym. Sci., 7, 685 (1951).

23. R. F. Boyer, Rubber Chem. Technol., 36, 1303 (1963).

24. J. Kopplemann and J. Gielssen, Z. Electrochem., 65, 689 (1961).

25. S. Saito, H. Sasabe, T. Nakajima, and K. Yada, J. Polym. Sci., Part A-2, 6, 1297 (1968).

26. G. Williams and D. C. Watts, Trans. Faraday Soc., 67, 1971 (1971).

27. M. H. Litt and A. V. Tobolsky, J. Macromol. Sci. Phys., B, 1, 433 (1967).

28. Y. Hotta, K. Tsuge, and Y. Wada, Repts. Progr. Polym. Phys. Japan, 8, 325 (1965).

29. Y. Wada, K. Tsuge, K. Arisawa, Y. Ohzawa, K. Shida, Y. Hotta, R. Hayakawa, and $T$. Nishi, J. Polym. Sci., Part C, 15, 101 (1966).

30. K. M. Sinnott, ibid., Part C, 14, 141 (1966).

31. T. Kakudate, F. Ishii, and T. Hideshima, Ab- stracts, SPSJ 20th Annual Meeting, Tokyo, May 27, 1971, p. 330.

32. H. Oberst, Kunststoffe, 53, 4 (1963).

33. Y. Wada and T. Kasahara, J. Appl. Polym. Sci., 11, 1661 (1967).

34. J. Heijboer, J. Polym. Sci., Part C, 16, 3755 (1968).

35. S. Matsuoka, C. J. Aloisio, and J. H. Daane, Appl. Polym. Symposia, 5, 103 (1967).

36. P. Hydemann and H. D. Guicking, Kolloid-Z. Z. Polym., 193, 16 (1963).

37. K. Nakamura, F. Hashimoto, M. Nakanishi, N. Kinjo, T. Kamatsu, and T. Nakagawa, in S. Onogi Ed., "Proceedings of the 5th International Congress on Rheology," Vol. 3, Kyoto, 1968, under the auspicies of The International Committee on Rheology, Universiry of Tokyo Press, Tokyo, 1970, p 409.

38. H. Kakutani and M. Asahina, J. Polym. Sci., Part A-2, 7, 1473 (1969). 